\title{
Endoplasmic reticulum stress, unfolded protein response and autophagy contribute to resistance to glucocorticoid treatment in human acute lymphoblastic leukaemia cells
}

\author{
SANGKAB SUDSAWARD ${ }^{1,2}$, SASIPRAPA KHUNCHAI ${ }^{2}$, CHUTAMAS THEPMALEE $^{3,4}$, AISHA OTHMAN ${ }^{1}$, \\ THAWORNCHAI LIMJINDAPORN ${ }^{4,5}$, PA-THAI YENCHITSOMANUS ${ }^{4}$, LUCIANO MUTTI ${ }^{6,7}$, \\ MARIJA KRSTIC-DEMONACOS ${ }^{1}$ and CONSTANTINOS DEMONACOS ${ }^{8}$
}

\author{
${ }^{1}$ School of Science, Engineering and Environment, University of Salford, Salford, M5 4WT, UK; \\ ${ }^{2}$ Department of Anatomy, Faculty of Medical Science, Naresuan University, Phitsanulok 65000; \\ ${ }^{3}$ Division of Biochemistry, School of Medical Science, University of Phayao, Phayao, 56000; \\ ${ }^{4}$ Division of Molecular Medicine, Department of Research; ${ }^{5}$ Department of Anatomy, Faculty of Medicine Siriraj Hospital, \\ Mahidol University, Bangkok 10700, Thailand; ${ }^{6}$ Sbarro Institute for Cancer Research and Molecular Medicine, \\ Center for Biotechnology, College of Science and Technology, Temple University, Philadelphia, PA 19122, USA; \\ ${ }^{7}$ Gruppo Italiano Mesotelioma, 15033 Casale Monferrato, Alessandria, Italy; ${ }^{8}$ Faculty of Biology Medicine and Health, \\ School of Health Science, Division of Pharmacy and Optometry, University of Manchester, Manchester, M13 9PT, UK
}

Received January 13, 2020; Accepted June 1, 2020

DOI: 10.3892/ijo.2020.5089

\begin{abstract}
Acute lymphoblastic leukaemia (ALL) is the most frequent childhood cancer and, although it is highly treatable, resistance to therapy, toxicity and side effects remain challenging. The synthetic glucocorticoid (GC) dexamethasone (Dex) is commonly used to treat ALL, the main drawback of which is the development of resistance to this treatment. The aim of the present study was to investigate potential molecular circuits mediating resistance and sensitivity to $\mathrm{GC}$-induced apoptosis in ALL. The leukaemia cell lines CEM-C7-14, CEM-C1-15 and MOLT4 treated with chloroquine (CLQ), thapsigargin (TG) and rotenone (ROT) were used to explore the roles of autophagy, endoplasmic reticulum (ER) stress/unfolded protein response (UPR) and reactive oxygen species (ROS) generation in the response to GC treatment. ROS levels were associated with increased cell death and mitochondrial membrane potential in rotenone-treated CEM cells. Autophagy inhibition by CLQ exhibited the strongest cytotoxic effect in GC-resistant leukaemia. Autophagy may act as a pro-survival mechanism in GC-resistant leukaemia since increasing trends in beclin-1 and microtubule-associated protein 1 light chain $3 \alpha$ levels were detected in CEM-C1-15
\end{abstract}

Correspondence to: Dr Constantinos Demonacos, Faculty of Biology Medicine and Health, School of Health Science, Division of Pharmacy and Optometry, University of Manchester, Stopford building, Oxford Road, Manchester, M13 9PT, UK

E-mail: constantinos.demonacos@manchester.ac.uk

Key words: acute lymphoblastic leukaemia, autophagy, endoplasmic reticulum stress, glucocorticoids, unfolded protein response and MOLT4 cells treated with Dex, whereas decreasing trends in these autophagy markers were observed in CEM-C7-14 cells. The intracellular protein levels of the ER stress markers glucose-regulated protein (GRP)78 and GRP94 were stimulated by Dex only in the GC-sensitive cells, suggesting a role of these chaperones in the GC-mediated ALL cell death. Increased cell surface levels of GRP94 were recorded in CEM-C7-14 cells treated with combination of Dex with TG compared with those in cells treated with TG alone, whereas decreasing trends were observed in CEM-C1-15 cells under these conditions. Taken together, the results of the present study demonstrated that autophagy may be a pro-survival mechanism in GC-resistant leukaemia, and by modulating intracellular and surface GRP94 protein levels, Dex is involved in the regulation of ER stress/UPR-dependent cell death and immune surveillance. These observations may be of clinical importance if confirmed in patients.

\section{Introduction}

Glucocorticoids (GCs) are steroid hormones produced by the adrenal glands in response to stress (1). Dexamethasone, a synthetic GC, has been used to treat patients suffering from numerous diseases such as leukaemia, inflammatory bowel disease, arthritis, asthma and allergies due to its regulatory role in cellular inflammatory responses, which include stimulating immunosuppression, inhibiting cell cycle progression and inducing apoptosis (2).

Acute lymphoblastic leukaemia (ALL) is a type of cancer of white blood cells that mainly affects children and teenagers (3). Several gene mutations and hazardous environmental factors (including radiation and toxic chemical exposure) are associated with ALL in children $(4,5)$. Factors affecting the 
development of resistance to ALL treatment include genetic variability in xenobiotic metabolism (6), prevalence of a GC receptor (GR) $\beta$ splicing variant (7), upregulation of the antiapoptotic Bcl-2 family proteins (8), differential phosphorylation of GR in Dex-induced apoptosis-resistant vs. sensitive ALL cells (9), alterations in DNA repair pathways (10) and cell cycle checkpoint defects (11).

Reactive oxygen species (ROS) serve an important role in the regulation of GR function (12) and the GC-mediated apoptosis in lymphoma cells (13). ROS mediate several cell signalling pathways (14) including cell cycle control (15), mitochondrial energy metabolism (16) and endoplasmic reticulum (ER) stress (17). ER stress is activated in rapidly proliferating cancer cells by the accumulation of misfolded or unfolded proteins as a consequence of high cellular ROS levels (18). The interplay among the mitochondria, ER and ROS levels, as well as the connection between ROS-mitochondrial membrane potential (MMP)-autophagy and ER stress have been extensively studied (19-24).

ER chaperone glucose-regulated protein (GRP)78 and GRP94 identify and bind to misfolded proteins, promote ER-associated protein degradation and contribute to the initiation of the immune response $(25,26)$. Induction of ER stress can lead to cell survival or death $(27,28)$. The pro-survival outcome of ER stress is in part due to the induction of its inositol-requiring enzyme $1 \mathrm{arm}$, which activates Jun N-terminal kinase (JNK) (29) and stimulates Bcl-2 phosphorylation to regulate autophagy through the modulation of the beclin-1 function and gene expression (30). In addition, diverse ER stress inducing conditions that involve the PKR-like endoplasmic reticulum kinase pathway and eukaryotic initiation factor $2 \alpha$ phosphorylation regulate the conversion of the pro-survival autophagy mediating microtubule-associated protein 1 light chain $3 \alpha$ (LC3) (31). However, if ER stress persists and the refolding of unfolded proteins is not resolved, unfolded protein response (UPR) ensues; UPR is primarily a pro-survival process, but prolonged stress may result in the induction of cell death $(27,28,32)$ via stimulation of CCAAT/enhancer-binding protein homologous protein (CHOP) (33).

The communication between the mitochondria and the endoplasmic reticulum (34) and the regulation of $\mathrm{Ca}^{2+}$ homeostasis by the integration of the function of Bcl-2 family members (35), endoplasmic reticulum chaperones (36) and their adjustment to the cellular redox state (37) is another mechanism controlling the pro-survival and pro-death pathways. Our bioinformatics analysis of $\mathrm{Ca}^{2+}$ signalling and autophagy pathways indicated that out of the 11,320 genes associated with $\mathrm{Ca}^{2+}$ signalling and 5,207 associated with autophagy, 1,109 genes are associated with both pathways (unpublished data). These genes include the inositol 1,4,5-trisphosphate receptor, ryanodine receptor, mitochondrial calcium uniporter, two pore segment channels, transient receptor potential channels, store-operated calcium channels, GRP78 and GRP94, the functions of which have been previously described (38-41).

T-cell ALL (T-ALL) and B-ALL differ in terms of prognostic factors and risk classification such as age, white blood cell count, hyperdiploidy and the presence of translocations $(42,43)$. Similar therapeutic schemes are used for the treatment of T-ALL and B-ALL, although significant toxicity associated with chemotherapy has been reported for B-ALL, as adults do not always benefit from therapeutic schemes used for paediatric patients $(42,43)$.

The present study aimed to investigate the molecular mechanisms mediating the resistance of T-ALL cells to Dex treatment. Established T-ALL CEM-C7-14 and CEM-C1-15 cell lines, which are sensitive and resistant, respectively, to Dex-induced apoptosis, were used to determine the crosstalk of multiple pathways involved in the response of T-ALL cells to chemotherapy. In particular, the potential consequences of the differential ROS detoxification in the T-ALL cells resistant or sensitive to Dex-induced apoptosis (44) and the ensuing changes in ER stress, autophagy and MMP were investigated. Emphasis was placed on understanding how changes in oxidative stress may be translated through autophagy, ER stress, UPR and the function of the ER chaperone proteins GRP94 and GRP78 to GC-mediated cell death.

\section{Materials and methods}

Cell culture. A previously established T-ALL in vitro system to study resistance to steroid response, consisting of the CEM-C1-15 and CEM-C7-14 cell lines (45) derived from lymphoblastic cells of a patient with ALL (46), was used in the present study. The T-ALL MOLT4 (cat. no. TCP-1010; ATCC) cells were used to explore whether the conclusions derived from the experiments using CEM cells were relevant to other types of T-ALL cells. Cells were cultured with $5 \% \mathrm{CO}_{2}$ at $37^{\circ} \mathrm{C}$ in RPMI-1640 medium (Sigma-Aldrich; Merck KGaA) supplemented with $10 \%$ foetal bovine serum (Sigma-Aldrich; Merck KGaA), $1 \%$ penicillin/streptomycin (Lonza Group,Ltd.) and 1\% L-glutamine (Lonza Group, Ltd.). Dextran-coated charcoal-treated serum (Sigma-Aldrich; Merck KGaA) was used in all experiments prior to the addition of drugs.

Drugs. The concentration of $1 \mu \mathrm{M}$ dexamethasone (Dex; Enzo Life Sciences, Inc.) was chosen for the treatment of the cells at the indicated time-points based on previous studies (47-49). The optimal treatment duration to observe differential effects of glucocorticoids in the sensitive vs. resistant cells was $48 \mathrm{~h}$ (49). The concentrations of chloroquine (CLQ; $100 \mu \mathrm{M}$; Sigma-Aldrich; Merck KGaA), thapsigargin (TG; $20 \mu \mathrm{M}$; Sigma-Aldrich; Merck KGaA) and rotenone (ROT; $20 \mu \mathrm{M}$; Sigma-Aldrich; Merck KGaA) were determined through optimization of experimental conditions (data not shown) and literature search $(47,48)$. All drugs were dissolved in dimethyl sulfoxide (chloroquine, thapsigargin and rotenone) or ethanol (dexamethasone) and used as indicated. The control groups were treated with DMSO.

Cell proliferation assay. CEM-C1-15, CEM-C7-14 and MOLT4 cells $\left(1 \times 10^{6}\right.$ cells $\left./ \mathrm{ml}\right)$ were seeded in 96 -well plates prior to drug treatment for $48 \mathrm{~h}$ at $37^{\circ} \mathrm{C}$. Once the drug incubation was complete, $20 \mu \mathrm{l}$ CellTiter 96 Aqueous MTS reagent working solution (cat. no. G1112; Promega Corporation) was added to the wells and incubated at $37^{\circ} \mathrm{C}$ for $4 \mathrm{~h}$. The absorbance of the samples was read at $490 \mathrm{~nm}$ using a microplate reader (Multiskan Ascent Thermo Labsystems 354 with Ascent software version 2.6) with $690 \mathrm{~nm}$ background compensation. 
MMP assay. CEM-C1-15, CEM-C7-14 and MOLT4 cells $\left(1 \times 10^{6}\right.$ cells $\left./ \mathrm{ml}\right)$ were seeded in 6-well plates prior to combination treatments (Dex, CLQ, TG, ROT, Dex + CLQ, Dex + TG or Dex + ROT) for $48 \mathrm{~h}$ at $37^{\circ} \mathrm{C}$. Subsequently, the cells were suspended in $1 \mathrm{ml}$ medium, and $6.25 \mu \mathrm{l}$ solution 7 (JC-1 dye; ChemoMetec $\mathrm{A} / \mathrm{S}$ ) was added to each sample, followed by 20 -min incubation at $37^{\circ} \mathrm{C}$. The stained cells were centrifuged at $400 \mathrm{x} \mathrm{g}$ for $5 \mathrm{~min}$ at room temperature, the supernatant was removed, and the samples were washed twice with PBS. The pellets were resuspended in $250 \mu 1$ solution 8 (DAPI), and the samples were analysed using a NucleoCounter NC-3000 with NucleoView software version 1 (ChemoMetec A/S).

ROS assay. A carboxy-H2DCADA probe (Invitrogen; Thermo Fisher Scientific, Inc.) was used to detect ROS generated in ALL cells treated with the aforementioned drugs. A probe containing 2',7'-dichlorofluorescein and calcein was used to detect oxidation by determination of the increase in fluorescence signalling using a flow cytometer, with excitation sources and filters for FITC channel. CEM-C1-15, CEM-C7-14 and MOLT4 cells $\left(1 \times 10^{6}\right.$ cells $\left./ \mathrm{ml}\right)$ were seeded in 6 -well plates before combination treatments (Dex, CLQ, TG, ROT, Dex + CLQ, Dex + TG or Dex + ROT) for $24 \mathrm{~h}$ at $37^{\circ} \mathrm{C}$. The cells were centrifuged at $753 \mathrm{x} \mathrm{g}$ for $3 \mathrm{~min}$ at $4^{\circ} \mathrm{C}$, and the supernatants were discarded. The cell pellets were resuspended in $1 \mathrm{ml}$ cold PBS and centrifuged at $753 \mathrm{x}$ g for $3 \mathrm{~min}$ at $4^{\circ} \mathrm{C}$. Carboxy-H2DCFDA dye $(100 \mu \mathrm{l})$ was added to the pellets, and the cells were incubated for $30-60 \mathrm{~min}$ at $37^{\circ} \mathrm{C}$ with $5 \% \mathrm{CO}_{2}$ in the dark. The dye was discarded by centrifuging the pellets at $753 \mathrm{x} \mathrm{g}$ for $3 \mathrm{~min}$ at $4^{\circ} \mathrm{C}$. The cells were washed with cold PBS and analysed by flow cytometry (BD FACSVerse ${ }^{\mathrm{TM}}$ with BD FACSuite software version 1.0; BD Biosciences) using the FITC channel.

Immunoblotting. CEM-C1-15, CEM-C7-14 and MOLT4 cells treated with Dex, CLQ, TG or ROT alone or a combination of Dex with CLQ, TG or ROT were harvested and lysed with RIPA buffer (cat. no. R0278; Sigma-Aldrich; Merck KGaA). Protein concentration was determined using the Bradford assay, and $40 \mu \mathrm{g}$ of total protein lysates were subjected to $12 \%$ sodium dodecyl sulphate polyacrylamide gel electrophoresis and transferred to a polyvinylidene difluoride membrane (EMD Millipore). Following blocking with 5\% skimmed milk in $1 \mathrm{X}$ PBS for $1 \mathrm{~h}$ at room temperature, the membrane was incubated with rabbit polyclonal anti-LC3A/B (cat. no. 4108; 1:1,000; Cell Signalling Technology, Inc.), rabbit monoclonal anti-beclin-1 (cat. no. 3495; 1:1,000; Cell Signalling Technology, Inc.), mouse monoclonal anti-GRP78 (cat. no. sc-376768; 1:500; Santa Cruz Biotechnology, Inc.), mouse monoclonal anti-GRP94 (cat. no. sc-53929; 1:500; Santa Cruz Biotechnology, Inc.) and rabbit polyclonal anti- $\beta$-actin (cat. no. ab8227; 1:2,000; Abcam) antibodies at $4^{\circ} \mathrm{C}$ overnight, followed by 1 -h incubation at $4^{\circ} \mathrm{C}$ with secondary antibodies conjugated with horseradish peroxidase (anti-rabbit cat. no. NA934; anti-mouse cat. no. NA931; 1:4,000; Cytiva). Protein bands were detected using an enhanced chemiluminescence solution (Thermo Fisher Scientific, Inc.) and an X-ray film (Fujifilm Corporation). The band intensities were estimated using ImageJ version 1.51 (National Institutes of Health).
Reverse transcription-quantitative PCR (RT-qPCR). Total RNAs were isolated from 3x106 CEM-C7-14, CEM-C1-15 and MOLT4 cells using a High Pure RNA Isolation kit (Roche Molecular Systems, Inc.) and the concentration of the total RNA for each sample was measured using a NanoDrop ${ }^{\mathrm{TM}}$ spectrophotometer (Thermo Fisher Scientific, Inc.). Complementary DNA was reverse-transcribed using a High-Capacity RNA-to-cDNA ${ }^{\mathrm{TM}}$ kit (Applied Biosystems; Thermo Fisher Scientific, Inc.) at $37^{\circ} \mathrm{C}$ for 1 h. QPCR was performed using GRP94, GRP78 and RPL19 (internal control) primers (Eurofins Scientific) mixed with SensiFAST SYBR ${ }^{\circledR}$ No-ROX kit (Bioline) as previously described $(50,51)$. The primer sequences were as follows: GRP78 forward, 5'-TGCCGTTCAAGGTGGTTG-3' and reverse, 5'-CCA AATAAGCCTCAGCGG-3'; GRP94 forward, 5'-CTGGGTCCA GCAGAAAAGAG-3' and reverse, 5'-CACTCCTTCCTTGGC AACAT-3'; RPL19 forward, 5'-ATGTATCACAGCCTGTAC CTG-3' and reverse, 5'-TTCTTGGTCTCTTCCTCCTTG-3'. The data were analysed using the $2^{-\Delta \Delta \mathrm{Cq}}$ method (52).

Cell surface staining assay. CEM-C1-15, CEM-C7-14 and MOLT4 cells $\left(1 \times 10^{6}\right.$ cells $\left./ \mathrm{ml}\right)$ were seeded in 6-well plates and combination treatments (Dex, CLQ, TG, ROT, Dex + CLQ, $\mathrm{Dx}+\mathrm{TG}$ or Dex + ROT) were applied for $48 \mathrm{~h}$ at $37^{\circ} \mathrm{C}$. Cells were washed with cold $\left(4^{\circ} \mathrm{C}\right) 2 \% \mathrm{FBS}$ in PBS before incubation with an anti-GRP94 antibody (9G10) conjugated to Alexa Fluor ${ }^{\circledR} 488$ (cat. no. sc-32249 AF488; Santa Cruz Biotechnology, Inc.) for $2 \mathrm{~h}$ at $4^{\circ} \mathrm{C}$. The cell pellet was fixed with $4 \%$ paraformaldehyde in FBS/PBS at $4^{\circ} \mathrm{C}$ for $15 \mathrm{~min}$, washed twice with $500 \mu \mathrm{l} \mathrm{PBS}\left(4^{\circ} \mathrm{C}\right)$ and flow cytometry (BD FACSVerse $^{\mathrm{TM}}$ with BD FACSuite software) was used to detect surface GRP94 fluorescence intensity.

Statistical analysis. Data are presented as the mean \pm standard error of the mean of data from three independent experiments. Statistical analysis was conducted using GraphPad Prism 8 Software (GraphPad Software, Inc.). One-way ANOVA and Dunnett's post hoc test were used for multiple comparisons. $\mathrm{P}<0.05$ was considered to indicate a statistically significant difference.

\section{Results}

Cytotoxic effects of individual and combined drug treatments. The present study analysed the autophagy pathway using CLQ, which is an autophagy inhibitor (53), ER stress using TG, which regulates $\mathrm{Ca}^{2+}$ homeostasis and activates ER stress (54), and ROS generation using ROT, which induces mitochondrial oxidative stress by inhibiting complex I of the mitochondrial respiratory chain and modulates autophagy (55) in GC-treated ALL cells. Time course and drug concentration studies were performed to determine the optimal experimental conditions (data not shown).

To assess ALL cell viability upon treatment with Dex, CLQ, TG and ROT alone or in combinations, MTS assay was performed in CEM-C7-14, CEM-C1-15 and MOLT4 cells treated with Dex alone or in combination with CLQ, TG or ROT (Fig. 1). Dex induced a less pronounced reduction in CEM-C1-15 cell viability compared with that of the CEM-C7-14 cells, whereas the viability of MOLT4 cells treated with Dex was $50 \%$ of that in untreated cells (Fig. 1). CLQ, TG and ROT 

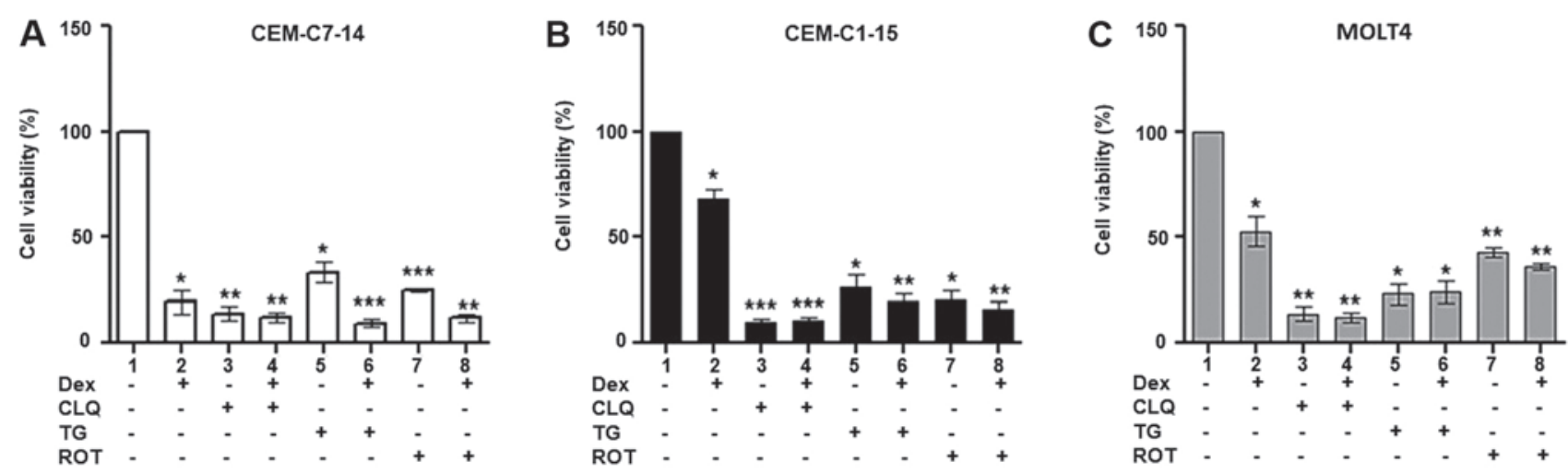

Figure 1. Cytotoxic effects of Dex and anticancer agents on ALL cells. Dex (10 $\mu \mathrm{M})$, CLQ (100 $\mu \mathrm{M})$, TG (20 $\mu \mathrm{M})$ and ROT (20 $\mu \mathrm{M})$ were used to treat (A) CEM-C7-14, (B) CEM-C1-15 and (C) MOLT4 cells for $48 \mathrm{~h}$ individually or in combination to assess the cell viability using MTS assay. Data are presented as the mean \pm SEM. ${ }^{*} \mathrm{P}<0.05,{ }^{* *} \mathrm{P}<0.01,{ }^{* * * *} \mathrm{P}<0.001$ vs. untreated control. ALL, acute lymphoblastic leukaemia; Dex, dexamethasone; CLQ, chloroquine; TG, thapsigargin; ROT, rotenone.
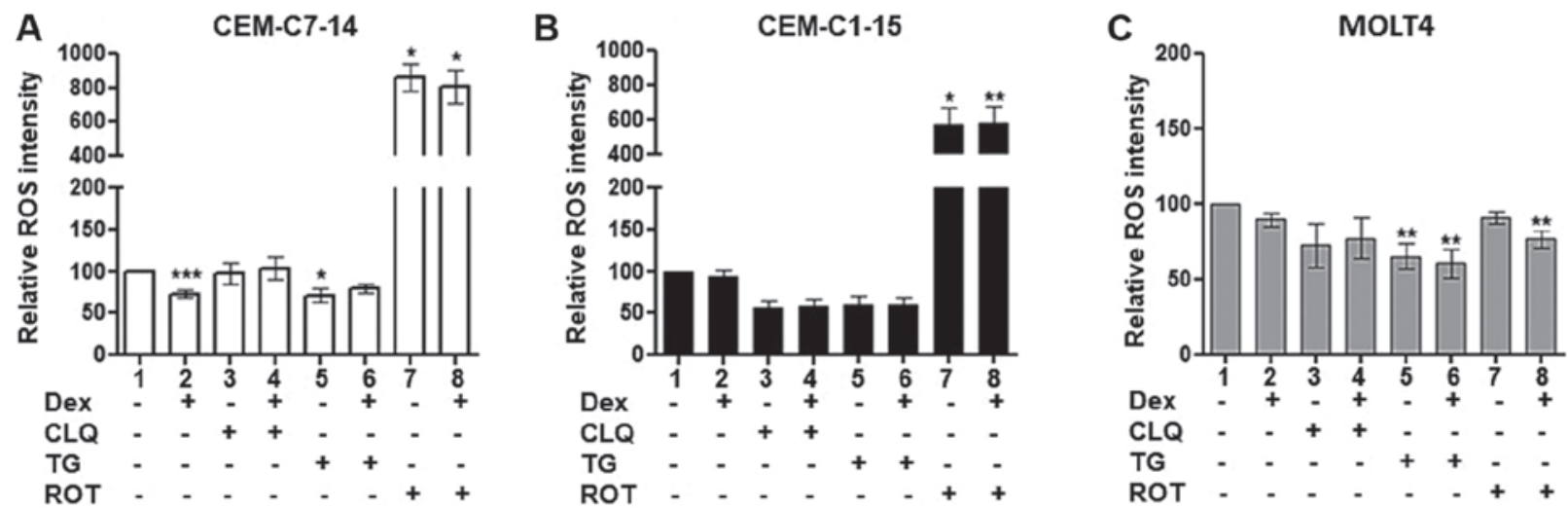

Figure 2. Effects of Dex and anticancer agents on ROS production. The relative ROS intensity was recorded in (A) CEM-C7-14, (B) CEM-C1-15 and (C) MOLT4 cells using a 2',7'-dichlorofluorescein probe and flow cytometry. Cells were treated with $1 \mu \mathrm{M}$ Dex, $50 \mu \mathrm{M} \mathrm{CLQ}, 10 \mu \mathrm{M}$ TG and $10 \mu \mathrm{M}$ ROT as indicated Data are presented as the mean $\pm \mathrm{SEM} .{ }^{*} \mathrm{P}<0.05,{ }^{* *} \mathrm{P}<0.01,{ }^{* * * *} \mathrm{P}<0.001$ vs. untreated control. Dex, dexamethasone; CLQ, chloroquine; TG, thapsigargin; ROT, rotenone; ROS, reactive oxygen species.

alone or in combination with Dex inhibited cell proliferation in all cells compared with that of untreated cells (Fig. 1). These results suggest that GCs alone mostly affected CEM-C7-14 cells, whereas CEM-C1-15 cells weakly responded to Dex, indicating a resistant phenotype, and MOLT4 cells appeared to exhibit a stronger response to Dex compared with CEM-C1-15 cells, but weaker compared with CEM-C7-14 cells (Fig. 1). A potent cytotoxic effect was observed in the CEM-C1-15 cells treated with CLQ alone or in combination with Dex (Fig. 1B). These results suggested that Dex treatment, autophagy inhibition, ER stress and induction of ROS generation exerted different cytotoxic effects in the studied ALL cells.

ROS generation in ALL cells treated with Dex, CLQ, TG and ROT alone or in combination. To investigate the potential involvement of ROS generation in response to GC treatment of ALL cells, CEM-C1-15, CEM-C7-14 and MOLT4 cells were treated with Dex, CLQ, TG or ROT alone or in combination, and the ROS levels were determined by flow cytometry. No significant difference in the ROS levels was observed in Dex-treated compared with untreated CEM-C1-15 cells (Figs. 2B and S1), whereas significantly decreased ROS levels were detected in Dex-treated compared with untreated
CEM-C7-14 cells (Figs. 2A and S1). No significant effect of Dex treatment on the ROS levels in MOLT4 cells was observed (Figs. 2C and S1). CLQ alone or in combination with Dex slightly decreased the ROS levels in CEM-C1-15 cells, although the changes were not statistically significant (Figs. 2B and S1). No significant effects of CLQ alone or in combination with Dex on the ROS levels of CEM-C7-14 and MOLT4 cells were observed (Figs. 2A, C and S1). Treatment of CEM-C7-14 cells with TG alone (Figs. 2A and S1) and of MOLT4 cells with TG alone or in combination with Dex (Figs. 2C and S1) significantly decreased ROS generation. By contrast, ROT alone or in combination with Dex substantially increased ROS production in CEM-C1-15 and CEM-C7-14 cells compared with that in the respective untreated cells (Figs. 2A and B and S1). This effect appeared to be more pronounced in CEM-C7-14 compared with CEM-C1-15 cells. The ROS levels were not affected in MOLT4 cells by ROT alone, whereas ROT-Dex co-treatment resulted in a significant decrease of ROS generation in these cells (Figs. 2C and S1).

In summary, lower ROS levels were recorded in Dexand TG-treated CEM-C7-14 cells compared with those in untreated cells, whereas CLQ had no effect. In MOLT4 cells, TG reduced the ROS levels. ROT increased the ROS levels 

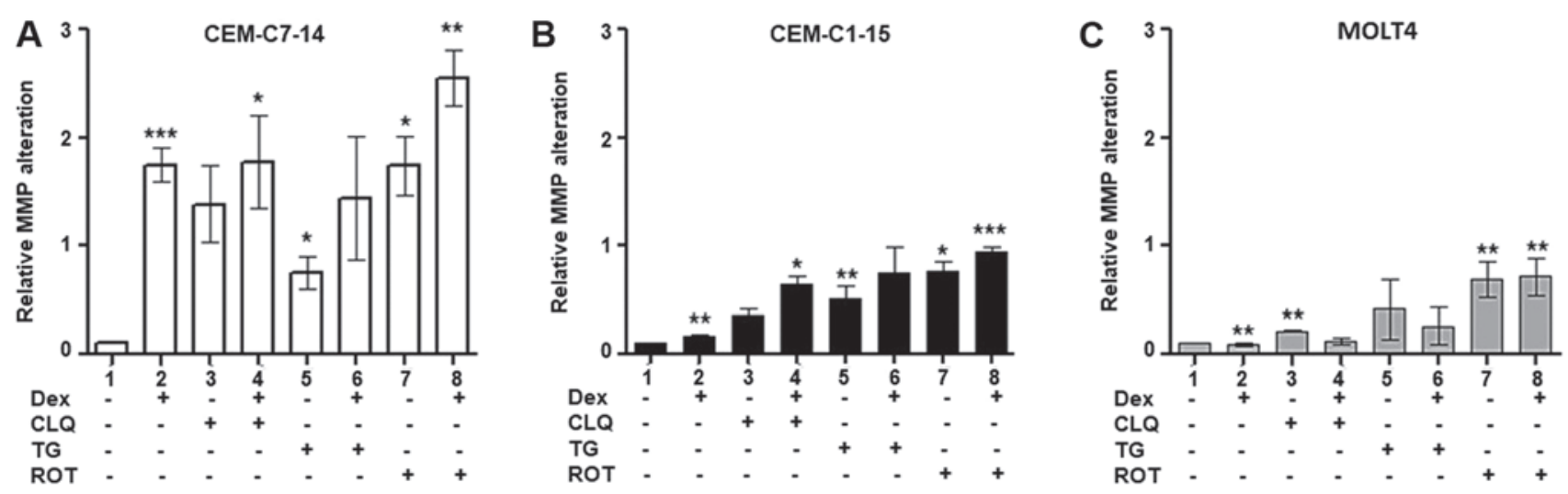

Figure 3. Effects of Dex and anticancer agents on MMP alteration. MMP was measured (A) in CEM-C7-14, (B) CEM-C1-15 and (C) MOLT4 cells treated with $1 \mu \mathrm{M}$ Dex, $50 \mu \mathrm{M}$ CLQ, $10 \mu \mathrm{M}$ TG and $10 \mu \mathrm{M}$ ROT for $48 \mathrm{~h}$ and using the JC-1 dye. Data are presented as the mean \pm SEM. "P<0.05, ${ }^{* *} \mathrm{P}<0.01$, ${ }^{* * * *} \mathrm{P}<0.001$ vs. untreated control. Dex, dexamethasone; CLQ, chloroquine; TG, thapsigargin; ROT, rotenone; MMP, mitochondrial membrane potential.

in CEM-C7-14 and CEM-C1-15 cells compared with those in the respective untreated cells, whereas it did not lead to an increase in the ROS levels in MOLT4 cells.

MMP in ALL cells treated with Dex, CLQ, TG and ROT alone or in combination. Considering the differential effects of individual Dex, CLQ, TG and ROT treatment on ROS generation in CEM-C7-14, CEM-C1-15 and MOLT4 cells, and the interplay between mitochondrial function and cellular ROS levels (56), the effects of Dex, CLQ, TG and ROT alone or combined with Dex on the MMP of ALL cells were investigated. Dex treatment alone significantly increased the MMP in CEM-C7-14 cells compared with that in untreated cells (Figs. 3A and S2A), whereas limited effects were observed in CEM-C1-15 and MOLT4 cells under the same conditions (Figs. 3B, C, S2B and C). Increased MMP was observed in the MOLT4 cell line treated with CLQ alone compared with that in untreated cells (Figs. 3C and S2C), as well as in CEM-C7-14 and CEM-C1-15 cells treated with the combination of CLQ and Dex compared with that in untreated cells (Figs. 3A, B, S2A and B). TG and ROT treatment alone increased MMP in CEM-C7-14 and CEM-C1-15 cells compared with that in untreated cells (Figs. 3A, B, S2A and B). Increased MMP was also observed in all studied cell lines treated with ROT or the combination of ROT and Dex compared with that in untreated cells (Figs. 3, S2A, B and C).

It appeared that most of the tested treatments increased MMP in CEM-C7-14 and CEM-C1-15 cells with higher effects in CEM-C7-14 cells, whereas in MOLT4 cells, CLQ and ROT increased MMP compared with that in untreated cells. Addition of Dex to TG-treated cells abolished the MMP increase in CEM-C7-14 and CEM-C1-15 cells.

Effects of individual and combined drug treatments on protein expression levels in ALL cells. To analyse the role of autophagy and ER stress in GC-mediated ALL cell survival or death, the protein levels of autophagy (beclin-1 and LC3-II) and ER stress (GRP78 and GRP94) indicators were analysed by western blotting in CEM-C7-14, CEM-C1-15 and MOLT4 cells (Figs. 4, S3A, B and C). A small but significant increase in beclin-1 protein levels, which mark the early stages of autophagy, was observed in TG-treated MOLT4 cells compared with untreated cells (Figs. 4A and S3B), whereas a decrease was observed in ROT and Dex co-treated CEM-C1-15 cells (Figs. 4A and S3C). LC3-II, is an indicator of late-stage autophagy, was increased in CEM-C1-15 and MOLT4 cells, but marginally decreased in CEM-C7-14 cells treated with Dex alone compared with the respective untreated cells (Figs. 4B, S3A, B and C). Higher LC3-II protein levels were observed in cells treated with CLQ and TG alone and in combination with Dex in all tested cell lines (Figs. 4B and S3B). No significant effects on LC3-II were observed in ROT-treated CEM-C7-14 and MOLT4 cells, whereas in CEM-C1-15 cells, downregulation of LC3-II was observed compared with untreated cells (Figs. 4B and S3C).

Dex treatment alone significantly increased GRP94 and GRP78 protein levels in CEM-C7-14 cells compared with those in untreated cells and there were no changes in CEM-C1-15 and MOLT4 cells (Figs. 4C, D and S3). A significant increase of GRP94 protein levels was also evident in CEM-C7-14 cells co-treated with Dex and CLQ or TG compared with those in untreated cells (Figs. 4C and S3A and B). In CEM-C1-15 cells treated with CLQ alone or with a combination of Dex and ROT and in MOLT4 cells treated with TG alone, increased levels of GRP94 were observed compared with those in untreated cells (Figs. 4C, S3A, B and C). Increased GRP78 protein levels were observed in CEM-C7-14 cells treated with Dex alone, ROT alone or a combination of CLQ or TG with Dex compared with those in untreated cells (Figs. 4D, S3A, B and C). The most substantial increase in both chaperone cellular levels was observed in GC-sensitive ALL cells (CEM-C7-14) co-treated with Dex and TG (Fig. S3B).

To determine whether the changes in GRP94 and GRP78 protein levels were at the gene expression level, the effects of Dex on GRP94 and GRP78 gene expression were studied using RT-qPCR. GRP94 and GRP78 mRNA levels were not affected in Dex-treated CEM cells, whereas decreased GRP94 mRNA levels were observed in MOLT4 cells compared with those in untreated cells (Fig. S4).

To summarise, compared with those in untreated cells, the levels of GRP94 and GRP78 markers of ER stress were increased in Dex-treated CEM-C7-14 cells, whereas the autophagy marker LC3-II was decreased in CEM-C7-14 and increased in CEM-C1-15 and MOLT4 cells. 

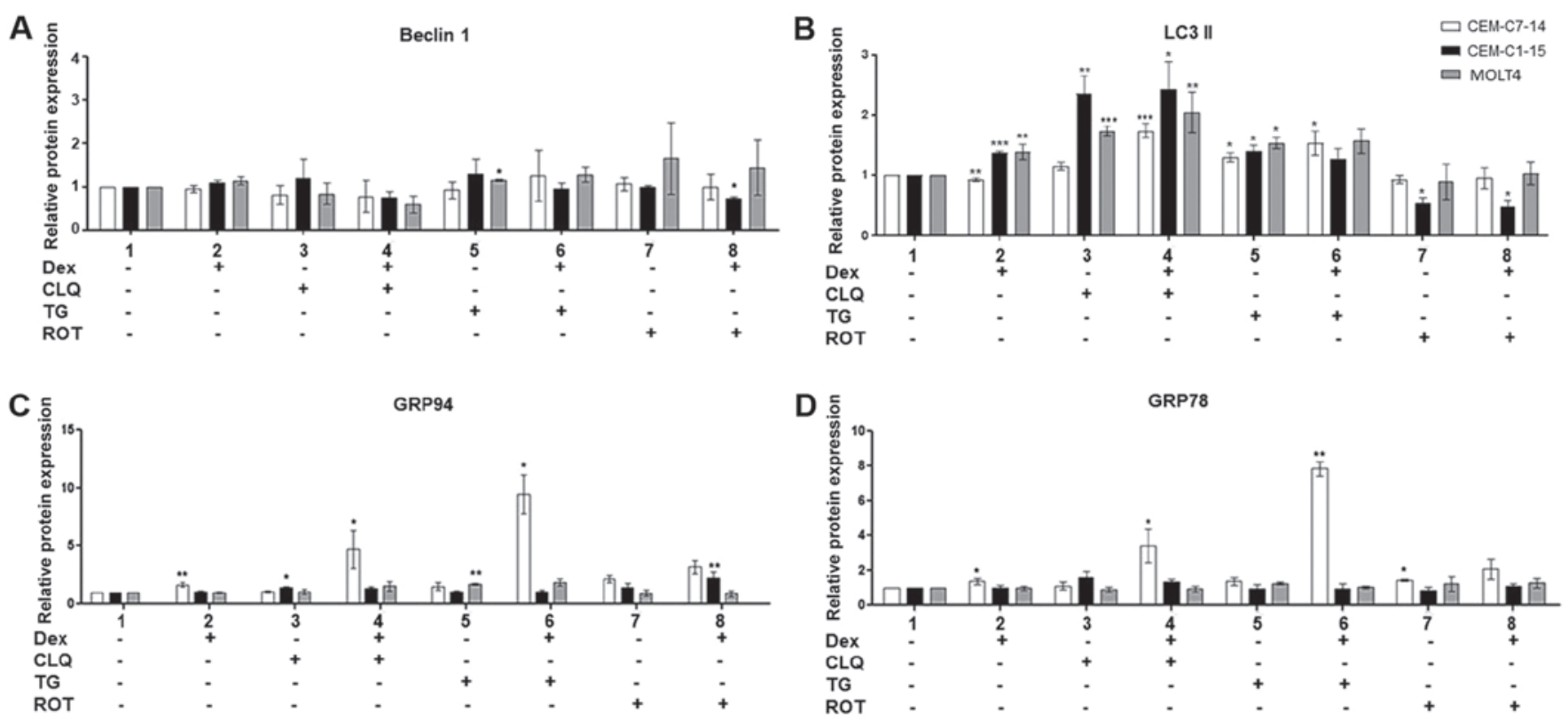

Figure 4. Protein expression in ALL following combination drug treatments. The protein levels of (A) beclin-1, (B) LC3-II, (C) GRP94 and (D) GRP78 were detected by western blotting in CEM-C7-14 (white bars), CEM-C1-15 (black bars) and MOLT4 (grey bars) cells after 48-h incubation with $1 \mu \mathrm{M}$ Dex, $50 \mu \mathrm{M}$ CLQ, $10 \mu \mathrm{M}$ TG and $10 \mu \mathrm{M}$ ROT individually or in combination as indicated. The values were normalised to the corresponding actin loading control. Data are presented as the mean \pm SEM. ${ }^{*} \mathrm{P}<0.05,{ }^{* * *} \mathrm{P}<0.01,{ }^{* * * *} \mathrm{P}<0.001$ vs. untreated control. ALL, acute lymphoblastic leukaemia; Dex, dexamethasone; CLQ, chloroquine; TG, thapsigargin; ROT, rotenone; LC3-II, microtubule-associated protein 1 light chain $3 \alpha$; GRP, glucose-regulated protein.

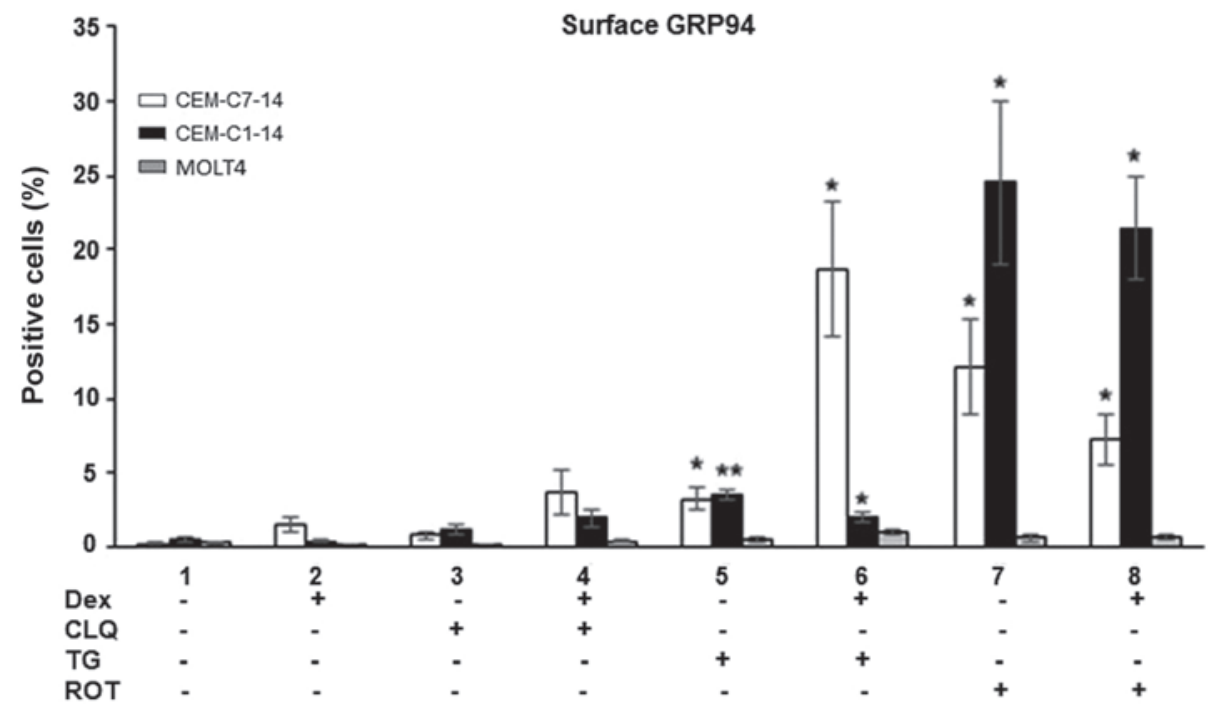

Figure 5. GRP94 surface expression levels on ALL cells. The CEM-C7-14 (white bars), CEM-C1-15 (black bars) and MOLT4 (grey bars) cells stained positive for GRP94 surface expression were detected by flow cytometry after 48-h treatments with $1 \mu \mathrm{M}$ Dex, $50 \mu \mathrm{M}$ CLQ, $10 \mu \mathrm{M}$ TG and $10 \mu \mathrm{M}$ ROT alone or in combination as indicated. Data are presented as the mean $\pm \mathrm{SEM} .{ }^{*} \mathrm{P}<0.05,{ }^{* *} \mathrm{P}<0.01$ vs. untreated control. ALL, acute lymphoblastic leukaemia; Dex, dexamethasone; CLQ, chloroquine; TG, thapsigargin; ROT, rotenone; GRP, glucose-regulated protein.

GRP94 surface expression in ALL. The extracellular surface GRP94 levels in ALL cells treated with Dex, CLQ, TG and ROT alone or in combination were investigated. TG and ROT treatment alone increased GRP94 surface levels in both the GC-sensitive and resistant CEM cells compared with those in the untreated control group (Figs. 5, S5A and B). Combination of TG and ROT with Dex increased the GRP94 surface levels in the CEM-C7-14 and CEM-C1-15 cells compared with those in untreated cells (Figs. 5, S5A and B). TG-Dex co-treatment exhibited a strong effect in CEM-C7-14 cells, whereas ROT effects individually or in combination with Dex was prominent in CEM-C1-15 cells. Surface GRP94 levels did not change in MOLT4 cells in any conditions (Figs. 5 and S5C).

These results suggested that GC or CLQ treatment alone did not significantly affect GRP94 surface location, whereas ER stress and ROS generation increased the GRP94 surface levels in CEM-C7-14 and CEM-C1-15 cells. Dex potentiated the effects of TG in CEM-C7-14 cells and inhibited TG effect in CEM-C1-15 cells, and ROT displayed more potent effects in the GC-resistant CEM-C1-15 cells compared with those in CEM-C7-14 cells. 


\section{Discussion}

The main therapeutic options for patients with ALL are based on treatment with GC hormones, which exert their effects by inducing apoptosis of malignant $\mathrm{T}$ cells through intrinsic and extrinsic pathways (50). As a result of greater potency and CNS penetration, dexamethasone is frequently selected as the treatment of choice for T-ALL $(42,43)$. A major drawback of $\mathrm{GC}$ treatment is the development of resistance (57). Inhibition of cell death and resistance of $\mathrm{T}$ cells to $\mathrm{GC}$ treatment has been attributed to a variety of molecular mechanisms (58), including autophagy (59). Autophagy is important for numerous physiological and pathological processes and for the function of immune system cells from antigen presentation to inflammatory signalling and metabolism (60). Various forms of autophagy triggered by distinct stimuli, such as ER stress, $\mathrm{Ca}^{2+}$ homeostasis and ROS signalling, control T cell survival (61). Since ER stress and UPR contribute to inflammatory signalling by activating a number of stress-responsive kinases including ERK, p38 mitogen-activated protein kinase and JNK (62), which have been demonstrated to induce GR post-translational modifications that affect its transcriptional activity $(51,63,64)$, it is possible that these mechanisms lead to resistance to GC-induced apoptosis in ALL by modulating the function of the GR $(51,62-64)$.

In the present study, Dex, CLQ, TG and ROT were used to treat GC-sensitive (CEM-C7-14) and resistant (CEM-C1-15) as well as MOLT4 cells to investigate the roles of autophagy, ER stress, UPR and oxidative stress in the mechanisms of GC-induced ALL apoptosis, development of resistance and potential ways to overcome it. Characterisation of the CEM genetic alterations has been reported in the literature $(47-49,65)$. Shedding light on this topic may facilitate the improvement of the current therapeutics and the development of novel anti-ALL treatments.

Autophagy has been reported to promote or suppress the proliferation of ALL cells (66). Recent studies have indicated that autophagy and ROS may serve a significant role in the determination of effects of GC treatment on ALL cells (59,67-69). Treatment of the GC-sensitive and resistant leukaemia cells with the autophagy inhibitor CLQ reduced their viability, and this reagent appeared to be the most effective in inducing GC- resistant ALL cell death. These findings suggested that autophagy may be involved in the GR-induced cell death (70) and it may facilitate the activation of the pro-survival mechanisms in GC-resistant cells, which was in agreement with previous studies indicating cytoprotective effects of autophagy in T-ALL cells treated with PI3K/mTOR or Akt inhibitors (71).

Accumulating evidence indicates an association between ROS generation and autophagy with alterations in the mitochondrial permeability transition pore (MPTP), ER stress, $\mathrm{Ca}^{2+}$ homeostasis and apoptosis $(20,72)$. In addition, the mitochondria-ER contact sites regulate immune cell survival/death decisions (73). The results of the present study demonstrated that GR-induced apoptosis in GC-sensitive ALL cells was associated with the increase in MMP disruption, suggesting that the regulation of the MPTP opening is a potential mechanism underlying the effects of Dex in the determination of ALL cell survival or death. A previous studies has demonstrated that spliced $\mathrm{x}$-box binding protein 1 and GRP78 are upregulated in $\mathrm{Ph}^{+}$leukaemia cell lines, leading to the activation of the UPR-related apoptosis protein CHOP (74). Although various cell type- and treatment duration-dependent effects were observed in the present study, definite conclusions regarding the chronological order of the occurrence of ROS generation, ER stress and autophagy in Dex-induced apoptosis-resistant vs. sensitive T-ALL cells cannot be drawn based on the experimental approaches used.

In the present study, Dex treatment of the GC-sensitive cells, but not the GC-resistant cells, led to increased expression levels of GRP78 and GRP94 compared with those in untreated cells. High GRP expression has been reported to be associated with cancer cell aggressiveness and metastatic potential in several cancer cell lines such as breast carcinoma, prostate adenocarcinoma, liver cancer, colorectal cancer, multiple myeloma and leukaemia (75). The results of the present study demonstrated that Dex stimulated GRP78 and GRP94 expression in GC-sensitive cells in a transcription-independent manner. GRP78 inhibits the translocation function on the ER membrane, preventing $\mathrm{Ca}^{2+}$ leakage from the ER to the cytoplasm (36) and altering the $\mathrm{Ca}^{2+}$-dependent mitochondrial apoptosis (73), which may explain the differential response of CEM-C7-14 and CEM-C1-15 cells to Dex treatment.

The results of the present study which indicated that Dex induced GRP94 expression in the GC-sensitive ALL cells were unexpected, as GRP78 and GRP94 upregulation is associated with a negative prognosis in various types of cancer such as prostate, oesophageal, gastric, breast and lung cancer (75). The role of GRP94 in the stimulation of T cells and regulation of immunity as well as induction of antitumour activity has been indicated (76). Surface localization of GRP94 is an important level of control of its function (77-79). In the present study, GCs induced the intracellular protein levels of these chaperones, but did not affect the mRNA or surface GRP94 expression levels; however, when Dex was combined with TG, the GRP94 surface expression increased in the GC-sensitive cells and decreased in the GC-resistant cells, suggesting that selective mechanisms determining the subcellular location of GRP94 may operate in the GC-resistant and sensitive cells. The molecular mechanisms involved in the regulation of resistance or sensitivity of ALL cells to Dex treatment through the induction of GRP94 are novel observations described in the present manuscript. In addition, considering the role of GRP proteins in the process of immunosurveillance and immune response $(26,80)$, it may be speculated that the changes occurring in the GRP levels and localization may directly or indirectly affect the immune response to the drug treatment. Therefore, it is possible that by differentially modulating the GRP94 surface levels in resistant and sensitive ALL cells co-treated with Dex and TG or ROT, GCs facilitate their recognition and elimination by the immune system, highlighting the need for further investigation of the secreted GRP levels and their effects on the immune system.

Limitations of the present study include the lack of an additional control cell line to consolidate the findings, and images demonstrating the morphology of the cells in culture were not provided. The effects of Dex alone or in combination with TG or ROT in B-ALL cells were not investigated, which should be addressed in the future. Of note, in vivo and in vitro drug doses are not comparable (81). Future in vivo studies with patients are required to verify the findings of the present study. 


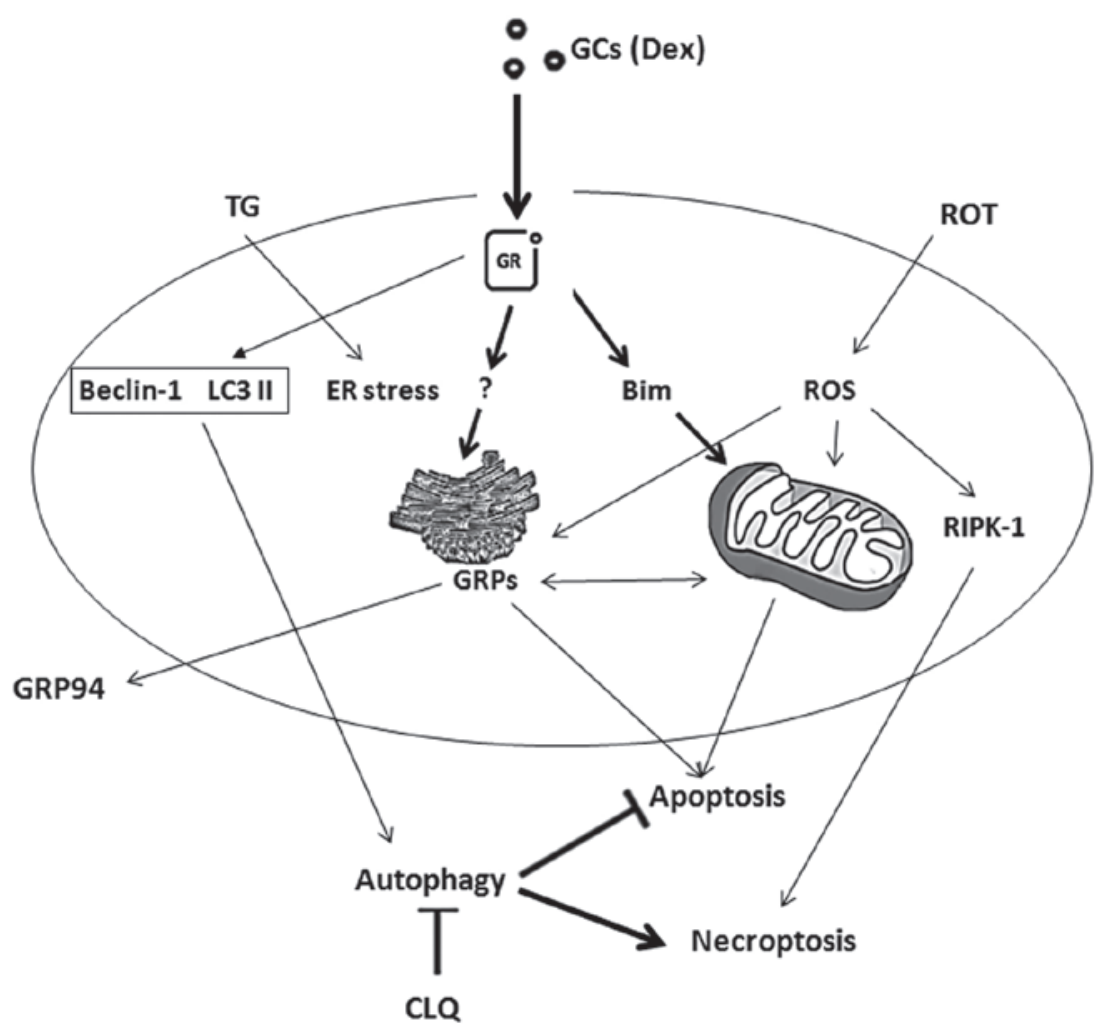

Figure 6. Summary of the hypothetical GC-mediated pathways leading to T-ALL cell death. GCs induce cell death in the sensitive CEM-C7-14 cells by inducing mitochondria and ER stress-mediated cell death through the induction of Bim and GRPs, respectively. Alteration of mitochondrial membrane potential may be involved in mitochondria-mediated apoptosis induced by ROT. GRP activation triggered by TG possibly regulates ER stress-mediated cell death CLQ-mediated inhibition of autophagy in CEM-C7-14 and CEM-C1-15 or induction of ROS generation by ROT in these cells may stimulate other types of cell death (e.g. necroptosis). ER stress and induction of ROS generation affect GRP94 plasma membrane relocalisation; the magnitude of this effect is differentially regulated in CEM-C7-14 and CEM-C1-15 and may be hormone-dependent. T-ALL, T-cell acute lymphoblastic leukaemia; GCs, glucocorticoids; GRP, glucose-regulated protein; Dex, dexamethasone; CLQ, chloroquine; TG, thapsigargin; ROT, rotenone; ROS, reactive oxygen species; ER, endoplasmic reticulum.

In conclusion, the drugs tested in the present study decreased the viability of CEM-C7-14 cells via mitochondria-ER communication mechanisms, upregulating GRP78 and GRP94 and inducing cell death (Fig. 6). These observations, if confirmed in clinical settings, may be used to increase the visibility of ALL cells to the immune system for the stratification of patients for immunotherapy and increased therapeutic success.

\section{Acknowledgements}

Part of this study was included in S. Sudsaward's PhD thesis.

\section{Funding}

This study was supported by the Royal Thai Government Scholarships, Ministry of Science and Technology, Thailand (to SS), the Staff Development Fund, Naresuan University (SK) the Thailand Research Fund (TRF; grant no. IRG5980006 to PY), the TRF-International Research Network (grant no. IRN58W001 to PY), the Siriraj Research Fund (grant no. R016034008 to PY), the Siriraj Chalermprakiat Grant (to PY and TL) and a TRF-Royal Golden Jubilee Ph.D. Scholarship (no. PHD/0044/2556 to CT).

\section{Availability of data and materials}

All data generated or analysed during this study are included in this published article.

\section{Authors' contributions}

SS planned and performed experiments, analysed the results and prepared the draft of the manuscript. SK, CT and AO performed the experiments, analysed the results and reviewed the manuscript. TL, PTY and LM analysed the results and reviewed the manuscript. MKD and CD formulated the hypothesis and supervised the study, interpreted the results and prepared the manuscript. All authors read and approved the final manuscript.

\section{Ethics approval and consent to participate}

Not applicable.

\section{Patient consent for publication}

Not applicable.

\section{Competing interests}

The authors declare that they have no competing interests.

\section{References}

1. Arlt W and Stewart PM: Adrenal corticosteroid biosynthesis, metabolism, and action. Endocrinol Metab Clin North Am 34: 293-313, 2005. 
2. Galon J, Franchimont D, Hiroi N, Frey G, Boettner A, Ehrhart-Bornstein M, O'Shea JJ, Chrousos GP and Bornstein SR: Gene profiling reveals unknown enhancing and suppressive actions of glucocorticoids on immune cells. FASEB J 16: 61-71, 2002.

3. Smith MA, Seibel NL, Altekruse SF, Ries LA, Melbert DL, O'Leary M, Smith FO and Reaman GH: Outcomes for children and adolescents with cancer: Challenges for the twenty-first century. J Clin Oncol 28: 2625-2634, 2010.

4. Greaves M: Infection, immune responses and the aetiology of childhood leukaemia. Nat Rev Cancer 6: 193-203, 2006.

5. Greaves MF and Wiemels J: Origins of chromosome translocations in childhood leukaemia. Nat Rev Cancer 3: 639-649, 2003.

6. Krajinovic M, Sinnett H, Richer C, Labuda D and Sinnett D Role of NQO1, MPO and CYP2E1 genetic polymorphisms in the susceptibility to childhood acute lymphoblastic leukemia. Int J Cancer 97: 230-236, 2002.

7. Webster JC, Oakley RH, Jewell CM and Cidlowski JA: Proinflammatory cytokines regulate human glucocorticoid receptor gene expression and lead to the accumulation of the dominant negative beta isoform: A mechanism for the generation of glucocorticoid resistance. Proc Natl Acad Sci USA 98: 6865-6870, 2001.

8. Alves NL, Derks IA, Berk E, Spijker R, van Lier RA and Eldering E: The Noxa/Mcl-1 axis regulates susceptibility to apoptosis under glucose limitation in dividing $\mathrm{T}$ cells Immunity 24: 703-716, 2006.

9. Berrou I, Demonacos C and Krstic-Demonacos M: Molecular mechanisms conferring resistance/sensitivity to glucocorticoid-induced apoptosis. In: Glucocorticoids - New Recognition of Our Familiar Friend: Qian X (ed). InTech, pp151-174, 2012.

10. Rosen DB, Putta S, Covey T, Huang YW, Nolan GP, Cesano A, Minden MD and Fantl WJ: Distinct patterns of DNA damage response and apoptosis correlate with Jak/Stat and PI3kinase response profiles in human acute myelogenous leukemia. PLoS One 5: e12405, 2010.

11. Healy J, Bélanger H, Beaulieu P, Larivière M, Labuda D and Sinnett D: Promoter SNPs in G1/S checkpoint regulators and their impact on the susceptibility to childhood leukemia. Blood 109: 683-692, 2007.

12. Nicolaides NC, Galata Z, Kino T, Chrousos GP and Charmandari E: The human glucocorticoid receptor: Molecular basis of biologic function. Steroids 75: 1-12, 2010.

13. Tome ME, Jaramillo MC and Briehl MM: Hydrogen peroxide signaling is required for glucocorticoid-induced apoptosis in lymphoma cells. Free Radic Biol Med 51: 2048-2059, 2011.

14. Zhang J, Wang X, Vikash V, Ye Q, Wu D, Liu Y and Dong W: ROS and ROS-mediated cellular signaling. Oxid Med Cell Longev 2016: 4350965, 2016.

15. Burhans WC and Heintz NH: The cell cycle is a redox cycle: Linking phase-specific targets to cell fate. Free Radic Biol Med 47: 1282-1293, 2009.

16. Liemburg-Apers DC, Willems PH, Koopman WJ and Grefte S: Interactions between mitochondrial reactive oxygen species and cellular glucose metabolism. Arch Toxicol 89: 1209-1226, 2015.

17. Tsiotra PC and Tsigos C: Stress, the endoplasmic reticulum, and insulin resistance. Ann NY Acad Sci 1083: 63-76, 2006.

18. Kato $\mathrm{H}$ and Nishitoh $\mathrm{H}$ : Stress responses from the endoplasmic reticulum in cancer. Front Oncol 5: 93, 2015.

19. Cao SS and Kaufman RJ: Endoplasmic reticulum stress and oxidative stress in cell fate decision and human disease. Antioxid Redox Signal 21: 396-413, 2014.

20. Zeeshan HM, Lee GH, Kim HR and Chae HJ: Endoplasmic reticulum stress and associated ROS. Int J Mol Sci 17: 327, 2016

21. Almanza A, Carlesso A, Chintha C, Creedican S, Doultsinos D Leuzzi B, Luís A, McCarthy N, Montibeller L, More S, et al: Endoplasmic reticulum stress signalling - from basic mechanisms to clinical applications. FEBS J 286: 241-278, 2019.

22. Llanos-González E, Henares-Chavarino ÁA, Pedrero-Prieto CM, García-Carpintero S, Frontiñán-Rubio J, Sancho-Bielsa FJ, Alcain FJ, Peinado JR, Rabanal-Ruíz Y and Durán-Prado M: Interplay between mitochondrial oxidative disorders and proteostasis in Alzheimer's disease. Front Neurosci 13: 1444, 2020

23. Delprat B, Crouzier L, Su TP and Maurice T: At the crossing of ER stress and MAMs: A key role of Sigma-1 receptor. In: Calcium Signaling. Islam MS (ed). Springer International Publishing, Cham, pp699-718, 2020.

24. Ferro F, Servais S, Besson P, Roger S, Dumas JF and Brisson L: Autophagy and mitophagy in cancer metabolic remodelling. Semin Cell Dev Biol 98: 129-138, 2020
25. Brown MK and Naidoo N: The endoplasmic reticulum stress response in aging and age-related diseases. Front Physiol 3: 263, 2012.

26. Binder RJ: Functions of heat shock proteins in pathways of the innate and adaptive immune system. J Immunol 193: 5765-5771, 2014.

27. Merksamer PI and Papa FR: The UPR and cell fate at a glance. J Cell Sci 123: 1003-1006, 2010.

28. Corazzari M, Gagliardi M, Fimia GM and Piacentini M: Endoplasmic reticulum stress, unfolded protein response, and cancer cell fate. Front Oncol 7: 78, 2017.

29. Ogata M, Hino S, Saito A, Morikawa K, Kondo S, Kanemoto S, Murakami T, Taniguchi M, Tanii I, Yoshinaga K, et al: Autophagy is activated for cell survival after endoplasmic reticulum stress. Mol Cell Biol 26: 9220-9231, 2006.

30. Wei Y, Pattingre S, Sinha S, Bassik M and Levine B: JNK1-mediated phosphorylation of Bcl-2 regulates starvation-induced autophagy. Mol Cell 30: 678-688, 2008

31. Kouroku Y, Fujita E, Tanida I, Ueno T, Isoai A, Kumagai H, Ogawa S, Kaufman RJ, Kominami E and Momoi T: ER stress (PERK/eIF2alpha phosphorylation) mediates the polyglutamine-induced LC3 conversion, an essential step for autophagy formation. Cell Death Differ 14: 230-239, 2007

32. Kharabi Masouleh B, Chevet E, Panse J, Jost E, O'Dwyer M, Bruemmendorf TH and Samali A: Drugging the unfolded protein response in acute leukemias. J Hematol Oncol 8: 87, 2015.

33. Sano R and Reed JC: ER stress-induced cell death mechanisms. Biochim Biophys Acta 1833: 3460-3470, 2013.

34. Csordás G, Weaver D and Hajnóczky G: Endoplasmic reticulum-mitochondrial contactology: Structure and signaling functions. Trends Cell Biol 28: 523-540, 2018.

35. Akl H, Vervloessem T, Kiviluoto S, Bittremieux M, Parys JB, De Smedt $\mathrm{H}$ and Bultynck G: A dual role for the anti-apoptotic Bcl-2 protein in cancer: Mitochondria versus endoplasmic reticulum. Biochim Biophys Acta 1843: 2240-2252, 2014.

36. Hammadi M, Oulidi A, Gackière $F$, Katsogiannou $M$, Slomianny C, Roudbaraki M, Dewailly E, Delcourt P, Lepage G, Lotteau S, et al: Modulation of ER stress and apoptosis by endoplasmic reticulum calcium leak via translocon during unfolded protein response: Involvement of GRP78. FASEB J 27: 1600-1609, 2013

37. Xia M, Zhang Y, Jin K, Lu Z, Zeng Z and Xiong W: Communication between mitochondria and other organelles: A brand-new perspective on mitochondria in cancer. Cell Biosci 9: 27, 2019.

38. Kondratskyi A, Kondratska K, Skryma R, Klionsky DJ and Prevarskaya N: Ion channels in the regulation of autophagy. Autophagy 14: 3-21, 2018

39. Sun F, Xu X, Wang X and Zhang B: Regulation of autophagy by $\mathrm{Ca}(2)$. Tumour Biol 37: 15467-15476, 2016.

40. Bootman MD, Chehab T, Bultynck G, Parys JB and Rietdorf K: The regulation of autophagy by calcium signals: Do we have a consensus? Cell Calcium 70: 32-46, 2018

41. Kania E, Pająk B and Orzechowski A: Calcium homeostasis and ER stress in control of autophagy in cancer cells. BioMed Res Int 2015: 352794, 2015.

42. Raetz EA and Teachey DT: T-cell acute lymphoblastic leukemia. Hematology Am Soc Hematol Educ Program 2016: 580-588, 2016.

43. Terwilliger $\mathrm{T}$ and Abdul-Hay M: Acute lymphoblastic leukemia: A comprehensive review and 2017 update. Blood Cancer J 7: e577, 2017.

44. Irwin ME, Rivera-Del Valle $\mathrm{N}$ and Chandra J: Redox control of leukemia: From molecular mechanisms to therapeutic opportunities. Antioxid Redox Signal 18: 1349-1383, 2013.

45. Harmon JM and Thompson EB: Isolation and characterization of dexamethasone-resistant mutants from human lymphoid cell line CEM-C7. Mol Cell Biol 1: 512-521, 1981.

46. Foley GE, Lazarus H, Farber S, Uzman BG, Boone BA and McCarthy RE: Continuous culture of human lymphoblasts from peripheral blood of a child with acute leukemia. Cancer 18: 522-529, 1965 .

47. Saenz GJ,Hovanessian R, Gisis AD and Medh RD: Glucocorticoidmediated co-regulation of RCAN1-1, E4BP4 and BIM in human leukemia cells susceptible to apoptosis. Biochem Biophys Res Commun 463: 1291-1296, 2015.

48. Jiang L, Xu L, Xie J, Li S, Guan Y, Zhang Y, Hou Z, Guo T, Shu X. Wang C, et al: Inhibition of autophagy overcomes glucocorticoid resistance in lymphoid malignant cells. Cancer Biol Ther 16: 466-476, 2015 
49. Norman MR and Thompson EB: Characterization of a glucocorticoid-sensitive human lymphoid cell line. Cancer Res 37: 3785-3791, 1977.

50. Lynch JT, Rajendran R, Xenaki G, Berrou I, Demonacos C and Krstic-Demonacos M: The role of glucocorticoid receptor phosphorylation in Mcl-1 and NOXA gene expression. Mol Cancer 9: 38,2010 .

51. Qattan MY, Bakker EY, Rajendran R, Chen DW, Saha V, Liu J, Zeef L, Schwartz JM, Mutti L, Demonacos C, et al: Differential regulation of cell death pathways by the microenvironment correlates with chemoresistance and survival in leukaemia. PLoS One 12: e0178606, 2017.

52. Livak KJ and Schmittgen TD: Analysis of relative gene expression data using real-time quantitative PCR and the $2^{-\Delta \Delta C T}$ method. Methods 25: 402-408, 2001.

53. Mauthe M, Orhon I, Rocchi C, Zhou X, Luhr M, Hijlkema KJ, Coppes RP, Engedal N, Mari M and Reggiori F: Chloroquine inhibits autophagic flux by decreasing autophagosome-lysosome fusion. Autophagy 14: 1435-1455, 2018.

54. Sehgal P, Szalai P, Olesen C, Praetorius HA, Nissen $P$ Christensen SB, Engedal N and Møller JV: Inhibition of the sarco/endoplasmic reticulum (ER) $\mathrm{Ca}^{2+}$-ATPase by thapsigargin analogs induces cell death via ER $\mathrm{Ca}^{2+}$ depletion and the unfolded protein response. J Biol Chem 292: 19656-19673, 2017.

55. Chen Y, McMillan-Ward E, Kong J, Israels SJ and Gibson SB Mitochondrial electron-transport-chain inhibitors of complexes I and II induce autophagic cell death mediated by reactive oxygen species. J Cell Sci 120: 4155-4166, 2007.

56. Zorova LD, Popkov VA, Plotnikov EY, Silachev DN, Pevzner IB, Jankauskas SS, Babenko VA, Zorov SD, Balakireva AV, Juhaszova M, et al: Mitochondrial membrane potential. Anal Biochem 552: 50-59, 2018.

57. Wilkinson L, Verhoog NJ and Louw A: Disease- and treatment-associated acquired glucocorticoid resistance. Endocr Connect 7: R328-R349, 2018

58. Bakker E, Qattan M, Mutti L, Demonacos C and KrsticDemonacos $\mathrm{M}$ : The role of microenvironment and immunity in drug response in leukemia. Biochim Biophys Acta 1863: 414-426, 2016.

59. Djavaheri-Mergny M, Giuriato S, Tschan MP and Humbert M: Therapeutic modulation of autophagy in leukaemia and lymphoma. Cells 8: E103, 2019.

60. Pua HH and He YW: Autophagy and lymphocyte homeostasis. Curr Top Microbiol Immunol 335: 85-105, 2009

61. Botbol Y, Guerrero-Ros I and Macian F: Key roles of autophagy in regulating T-cell function. Eur J Immunol 46: 1326-1334, 2016.

62. Hotamisligil GS and Davis RJ: Cell signaling and stress responses. Cold Spring Harb Perspect Biol 8: a006072, 2016.

63. Davies L, Karthikeyan N, Lynch JT, Sial EA, Gkourtsa A, Demonacos C and Krstic-Demonacos M: Cross talk of signaling pathways in the regulation of the glucocorticoid receptor function. Mol Endocrinol 22: 1331-1344, 2008.

64. Chen DW, Saha V, Liu JZ, Schwartz JM and Krstic-Demonacos M Erg and AP-1 as determinants of glucocorticoid response in acute lymphoblastic leukemia. Oncogene 32: 3039-3048, 2013.

65. Medh RD, Webb MS, Miller AL, Johnson BH, Fofanov Y, Li T, Wood TG, Luxon BA and Thompson EB: Gene expression profile of human lymphoid CEM cells sensitive and resistant to glucocorticoid-evoked apoptosis. Genomics 81: 543-555, 2003.
66. Evangelisti C, Evangelisti C, Chiarini F, Lonetti A, Buontempo F, Neri LM, McCubrey JA and Martelli AM: Autophagy in acute leukemias: A double-edged sword with important therapeutic implications. Biochim Biophys Acta 1853: 14-26, 2015.

67. Auberger P and Puissant A: Autophagy, a key mechanism of oncogenesis and resistance in leukemia. Blood 129: 547-552, 2017.

68. Takahashi H, Inoue J, Sakaguchi K, Takagi M, Mizutani S and Inazawa J: Autophagy is required for cell survival under L-asparaginase-induced metabolic stress in acute lymphoblastic leukemia cells. Oncogene 36: 4267-4276, 2017.

69. Rothe K, Porter V and Jiang X: Current outlook on autophagy in human leukemia: Foe in cancer stem cells and drug resistance, friend in new therapeutic interventions. Int J Mol Sci 20: E461, 2019.

70. Laane E, Tamm KP, Buentke E, Ito K, Kharaziha P, Oscarsson J, Corcoran M, Björklund AC, Hultenby K, Lundin J, et al: Cell death induced by dexamethasone in lymphoid leukemia is mediated through initiation of autophagy. Cell Death Differ 16: 1018-1029, 2009

71. Simioni C, Neri LM, Tabellini G, Ricci F, Bressanin D, Chiarini F, Evangelisti C, Cani A, Tazzari PL, Melchionda F, et al: Cytotoxic activity of the novel Akt inhibitor, MK-2206, in T-cell acute lymphoblastic leukemia. Leukemia 26: 2336-2342, 2012.

72. Verfaillie T, Salazar M, Velasco G, Agostinis P and Linking ER Linking ER stress to autophagy: Potential implications for cancer therapy. Int J Cell Biol 2010: 930509, 2010.

73. Martinvalet D: The role of the mitochondria and the endoplasmic reticulum contact sites in the development of the immune responses. Cell Death Dis 9: 336, 2018.

74. Dengler MA, Staiger AM, Gutekunst M,Hofmann U,Doszczak M, Scheurich P, Schwab M, Aulitzky WE and van der Kuip H: Oncogenic stress induced by acute hyper-activation of Bcr-Abl leads to cell death upon induction of excessive aerobic glycolysis. PLoS One 6: e25139, 2011.

75. Lee AS: Glucose-regulated proteins in cancer: Molecular mechanisms and therapeutic potential. Nat Rev Cancer 14: 263-276, 2014.

76. Biswas C, Sriram U, Ciric B, Ostrovsky O, Gallucci S and Argon Y: The N-terminal fragment of GRP94 is sufficient for peptide presentation via professional antigen-presenting cells. Int Immunol 18: 1147-1157, 2006.

77. Ansa-Addo EA, Thaxton J, Hong F, Wu BX, Zhang Y, Fugle CW, Metelli A, Riesenberg B, Williams K, Gewirth DT, et al: Clients and oncogenic roles of molecular chaperone gp96/grp94. Curr Top Med Chem 16: 2765-2778, 2016.

78. Duan XF and Xin YW: Overexpression of molecule GRP94 favors tumor progression in lung adenocarcinoma by interaction with regulatory T cells. Thorac Cancer 11: 704-712, 2020.

79. Wiersma VR, Michalak M, Abdullah TM, Bremer E and Eggleton P: Mechanisms of translocation of ER chaperones to the cell surface and immunomodulatory roles in cancer and autoimmunity. Front Oncol 5: 7, 2015.

80. Binder RJ. Immunosurveillance of cancer and the heat shock protein-CD91 pathway. Cell Immunol 343: 103814, 2019.

81. Sundman-Engberg B, Tidefelt U, Gruber A and Paul C: Intracellular concentrations of mitoxantrone in leukemic cells in vitro vs. in vivo. Leuk Res 17: 347-52, 1993. 